\title{
TUBO GÁSTRICO ISOPERISTÁLTICO NO TRATAMENTO PALIATIVO DO CARCINOMA IRRESSECÁVEL DO ESÔFAGO
}

\author{
GASTRO-ESOPHAGEAL ISOPERISTALTIC BYPASS AS PALLIATIVE TREATMENT \\ OF THE IRRESECTABLE ESOPHAGEAL CANCER
}

\author{
Alexandre Cruz Henriques, TCBC-SP ${ }^{1}$ \\ Sérgio Pezzolo, TCBC-SP ${ }^{1}$ \\ Marise Gomes Faure, ACBC-SP ${ }^{2}$ \\ Luís Teodoro da Luz, ACBC-SP2 \\ Carlos Alberto Godinho ${ }^{2}$ \\ Manlio Basilio Speranzini, TCBC-SP ${ }^{3}$
}

\begin{abstract}
RESUMO: A meta do tratamento dos pacientes portadores de carcinoma avançado de esôfago é o alívio da disfagia. Objetivo: O objetivo deste trabalho é relatar a experiência dos autores na utilização da derivação esofagogástrica através de um tubo gástrico isoperistáltico seguido de radioterapia, no tratamento de doentes portadores de carcinoma irressecável do esôfago. Método: No período de 1990 a 1999, 30 pacientes foram submetidos à cirurgia de derivação. Vinte e quatro doentes (80\%) eram do sexo masculino e seis (20\%) do feminino; a idade variou de 27 a 69 anos, média de 49,3 anos. Em todos os casos o diagnóstico foi confirmado por endoscopia digestiva alta e biópsia. Aqueles com tumores maiores que $6 \mathrm{~cm}$ ao esofagograma ou com sinais de invasão da árvore respiratória à broncoscopia foram considerados irressecáveis. Após avaliação clínica e preparo pré-operatório foram submetidos à operação de derivação. $\mathrm{O}$ ato operatório foi realizado por duas equipes, uma na região cervical e outra na abdominal e a duração da intervenção variou entre três a quatro horas. Após a alta hospitalar os pacientes foram encaminhados para a radioterapia. Resultados: Não houve óbito operatório. A mortalidade pósoperatória foi de $10 \%$, um caso de tromboembolismo pulmonar e dois de broncopneumonia. Treze pacientes $(43,3 \%)$ desenvolveram fístula cervical e em 11 ocorreu o fechamento espontâneo da fístula; um caso necessitou de reoperação e outro veio a falecer no $14^{\circ}$ dia pós-operatório com a fístula aberta. Oito pacientes $(26,6 \%)$ apresentaram estenose da anastomose esôfago-tubo; todos evoluíram bem com dilatação endoscópica. A deglutição foi restabelecida em todos os pacientes até o momento do óbito, excetuando aqueles que faleceram em virtude de complicações pós-operatórias (três casos). O tempo de internação variou de 12 a 45 dias e a sobrevida média foi de 7,9 meses. Conclusões: Os autores concluíram que nos pacientes portadores de carcinoma irressecável do esôfago, a derivação da obstrução através de um tubo gástrico isoperistáltico atinge seu objetivo primeiro que é aliviar a disfagia até o momento do óbito. A morbidade é alta, porém a maioria das complicações tem evolução benigna. A mortalidade é aceitável levando-se em conta a gravidade do carcinoma do esôfago.
\end{abstract}

Descritores: Carcinoma do esôfago; Tubo gástrico isoperistáltico; Cirurgia paliativa.

\section{INTRODUÇÃO}

Não houve melhora significativa, nos últimos 40 anos, da taxa de cura oncológica dos pacientes portado- res de carcinoma de esôfago ${ }^{1}$. No Brasil o diagnóstico desta afecção continua sendo feito quando há pouca possibilidade de tratamento curativo². A disfagia é o principal sintoma e a responsável pela desnutrição grave; devi-

1. Professor Assistente do Departamento de Cirurgia da Faculdade de Medicina do ABC.

2. Cirurgião Colaborador do Departamento de Cirurgia da Faculdade de Medicina do ABC.

3. Professor Titular da Disciplina de Cirurgia do Aparelho Digestivo da Faculdade de Medicina do ABC.

Recebido em 29/09/2000

Aceito para publicação em 05/07/2001

Trabalho realizado no Serviço de Cirurgia do Aparelho Digestivo do Hospital de Ensino da Faculdade de Medicina do $\mathrm{ABC}$. 
do à obstrução esofágica, complicações pulmonares são freqüentes ${ }^{3}$. Diante deste quadro, a principal meta do tratamento é obter alívio da disfagia com mínima morbimortalidade ${ }^{3}$. Quando o tumor é ressecável, o tratamento preconizado é a esofagectomia subtotal mesmo com intenção paliativa. O tratamento dos tumores irressecáveis é assunto aberto, e comporta distintas abordagens: ablação térmica (terapia com laser), radioterapia, próteses transtumorais e diversos procedimentos cirúrgicos com intenção de abrir uma via de alimentação através de um estoma ou de uma derivação.

A utilização do laser propicia a recanalização da luz esofágica na maioria dos casos, com baixa morbidade $(1,5 \%$ a $18 \%$ ) e mortalidade $(0 \%$ a $2 \%)$, porém apenas $80 \%$ dos pacientes conseguem alimentar-se adequadamente por via oral, mesmo com o esôfago desobstruído; a necessidade de sessões repetidas e o fato de ser método restrito a poucos centros, representam desvantagens adicionais ${ }^{2,4-6}$.

Altas doses de radioterapia permitem melhora transitória da disfagia em 50\% a 70\% dos pacientes que completam o tratamento, o que não ocorre em $20 \%$ dos ca$\operatorname{sos}^{3,7}$. Estenoses esofágicas (26\%), fístulas esofagotraqueais (5\%) e hemorragia profusa (3\%) são complicações da radioterapia que pioram a condição clínica do paciente $^{6}$.

A utilização de próteses apresenta 5\% a 30\% de complicações imediatas (perfurações e sangramento), $7 \%$ a $34 \%$ de complicações tardias (migração e obstrução) e mortalidade que varia entre $1,5 \%$ a $16 \%{ }^{6,8-10}$. O desenvolvimento das próteses auto-expansivas representa um avanço desta modalidade terapêutica, porém persistem 3\% de mortalidade e $30 \%$ de morbidade (sensação persistente de corpo estranho no esôfago, incapacidade de ingestão de determinados tipos de alimentos, obstrução e migração) $)^{6,11}$.

A gastrostomia é hoje citada apenas para ser condenada $^{12}$. Embora seja de fácil execução não traz qualquer alívio da disfagia, não impede a aspiração de saliva para a árvore respiratória e castiga o paciente pela presença da sonda fixa à parede abdominal que, com frequiência, apresenta vazamento do conteúdo gástrico ao seu redor. Além disso, deve-se ressaltar as seguintes complicações: deiscência de parede, evisceração, hemorragia ao redor da sonda, obstrução e perda da sonda ${ }^{6,13,14}$.

A obstrução esofágica pode ser transposta de diversas maneiras, utilizando-se o estômago, o jejuno ou o cólon ${ }^{6}$. Estas técnicas apresentam morbi-mortalidade significativas e nenhuma delas ganhou aceitação universal.

A existência de várias modalidades terapêuticas demonstra, por si só, que nenhuma delas é a ideal. Dentro deste panorama sombrio, o tubo gástrico isoperistáltico foi desenvolvido com o intuito de resolver a disfagia dos pacientes portadores de carcinoma avançado do esôfago, com um procedimento que pretende ser simples, com baixa morbi-mortalidade e com baixo custo. Nos países em desenvolvimento, a derivação cirúrgica permanece como uma opção relevante diante do alto custo das próteses auto-expansivas e da terapia com laser $^{15}$. Embora o custo global da terapêutica cirúrgica seja maior que o da terapêutica endoscópica com próteses auto-expansivas, o custo do pro- cedimento é significativamente maior na terapêutica endoscópica devido ao alto custo da endoprótese ${ }^{16}$, fato que inviabiliza esta modalidade de tratamento na maioria dos hospitais públicos brasileiros ${ }^{16}$. Estudo comparativo do tratamento paliativo do carcinoma avançado do esôfago com tubo gástrico isoperistáltico ou com prótese metálica auto-expansiva demonstrou que as duas modalidades terapêuticas permitiram igualmente a restauração da alimentação oral e que a ocorrência de complicações foi quantitativamente semelhante nos dois grupos ${ }^{16}$.

A partir de 1990, os pacientes com neoplasia avançada do esôfago atendidos em nosso serviço, passaram a ser tratados segundo um protocolo: o objetivo deste trabalho é relatar a nossa experiência na utilização da derivação esofagogástrica através de um tubo gástrico isoperistáltico seguido de radioterapia, no tratamento de portadores de carcinoma irressecável do esôfago.

\section{MÉTODO}

No período de janeiro de 1990 a janeiro de 1998, 30 pacientes portadores de carcinoma irressecável de esôfago, estádio III e IV, foram submetidos à derivação esofagogástrica através da confecção de um tubo gástrico isoperistáltico, no Hospital de Ensino da Faculdade de Medicina do ABC. Vinte e quatro pacientes $(80 \%)$ eram do sexo masculino e seis $(20 \%)$ do sexo feminino, com idade variando de 27 a 69 anos, média de 49,3 anos.

Disfagia e emagrecimento foram os sintomas mais freqüentes, presentes em todos os pacientes, seguidos de odinofagia em 17 pacientes $(56,6 \%)$, sialorréia em oito $(26,6 \%)$ e regurgitação em oito $(26,6 \%)$. O diagnóstico foi confirmado pela radiografia contrastada de esôfago, endoscopia digestiva alta e biópsia; em 28 casos $(93,3 \%)$ a histopatologia evidenciou carcinoma espinocelular e em dois $(6,7 \%)$ adenocarcinoma.

A lesão localizava-se no terço superior em dois pacientes $(6,7 \%)$, no terço médio em $22(73,4 \%)$ e no terço inferior em seis (20\%). Em todos os pacientes a lesão era maior que $8 \mathrm{~cm}$. O estadiamento constava do exame físico, radiografia de tórax, ultra-sonografia abdominal e broncoscopia. Tomografia computadorizada do tórax foi realizada a partir de 1997, quando a instituição adquiriu o equipamento. A ultra-sonografia evidenciou metástases hepáticas em quatro pacientes $(12 \%)$ e a broncoscopia revelou abaulamento na região da carina em três pacientes $(10 \%)$.

Foram considerados irressecáveis os tumores com mais de $6 \mathrm{~cm}$ ao esofagograma ou aqueles que abaulavam ou infiltravam a árvore respiratória.

Todos os pacientes foram submetidos à avaliação clínica pré operatória: 27 pacientes $(90 \%)$ apresentavam desnutrição com perda superior a $20 \%$ do peso habitual, $17(56,6 \%)$ anemia, seis (20\%) hipertensão arterial sistêmica, seis $(20 \%)$ doença pulmonar obstrutiva crônica e dois $(6,6 \%)$ diabetes mellitus. Em dois pacientes, a radiografia mostrava seqüela de tuberculose no pulmão direito, em um coexistia megaesôfago grau III e em outro, colecistopatia crônica calculosa assintomática. Infecção 
pulmonar no período pré-operatório foi diagnosticada em quatro pacientes $(13,3 \%)$ e tratada com antibioticoterapia sistêmica; o ato cirúrgico só foi realizado após regressão completa do quadro. Foram excluídos pacientes com doença sistêmica grave, muito depauperados ou com sinais de disseminação maciça (caquexia, ascite, carcinomatose etc.).

Durante a execução da endoscopia, sempre que o tumor fosse transponível ao aparelho, o endoscopista instalava uma sonda de Dobbhoff no estômago para introdução de dietas. Nutrição parenteral por sete a dez dias foi utilizada nos casos com obstrução intransponível do esôfago. A todos permitiu-se ingestão voluntária de dieta líquida. Fisioterapia respiratória foi sistematicamente realizada no período pré-operatório. A profilaxia dos fenômenos tromboembólicos foi feita com administração de fraxiparina, na dose de 40mg por dia, por via subcutânea, iniciada 12 horas antes da cirurgia e mantida por cinco a sete ou até a deambulação.

Os procedimentos cirúrgicos foram realizados com duas equipes, uma na região abdominal e outra na cervical. O acesso abdominal se fazia através de laparotomia mediana xifoumbilical. Após inventário da cavidade abdominal, o tubo gástrico isoperistáltico era confeccionado liberando-se amplamente toda a grande curvatura, inclusive o fundo gástrico; a grande curvatura era seccionada a partir do seu fundo, com grampeador linear cortante de $75 \mathrm{~mm}$. Este tubo nutrido pela artéria gastroepiplóica direita, era realizado de tal modo a ter $4 \mathrm{~cm}$ de largura, iniciando-se próximo à cárdia e estendendo-se até cerca de $2 \mathrm{~cm}$ do piloro; em média tinha 35 a $40 \mathrm{~cm}$ de comprimento (Figura 1). Um segundo plano de sutura contínua com fio de poliglecaprone 0000 era feito para invaginação da sutura mecânica. $\mathrm{O}$ acesso ao esôfago cervical era feito por cervicotomia esquerda ao longo da margem anterior do músculo esternoclidomastóideo. O esôfago e o nervo laríngeo recorrente eram dissecados e isolados; o esôfago era seccionado e seu coto distal fechado; após a ascensão do tubo à região cervical por um túnel retroesternal previamente dissecado, o coto proximal do esôfago era anastomosado ao tubo, de maneira terminoterminal, em plano único, com fio de poliglecaprone 0000 (total no esôfago e seromuscular no estômago). Um cateter nasoenteral de Dobbhoff era introduzido até a primeira porção duodenal para nutrição enteral. As duas vias de acesso eram, a seguir, fechadas, drenando-se a região cervical com dreno de Penrose. O tempo cirúrgico variou entre três e quatro horas.

O período pós-operatório imediato era realizado na unidade de terapia intensiva. Os pacientes recebiam antibioticoprofilaxia (cefoxitina), iniciada na indução anestésica, durante 24 horas. Fisioterapia respiratória era iniciada precocemente e dieta enteral pela sonda de Dobbhoff era introduzida após a resolução do íleo adinâmico; dieta por via oral era iniciada, geralmente no $10^{\circ}$ dia pós-operatório, quando não havia sinais de fístula esofagocutânea (após teste de ingestão de solução de azul de metileno). O dreno de Penrose cervical era, geralmente, retirado no quinto dia pós-operatório. Na presença de fístula esofagocutânea, alguns pontos eram retirados do terço inferior da inci-

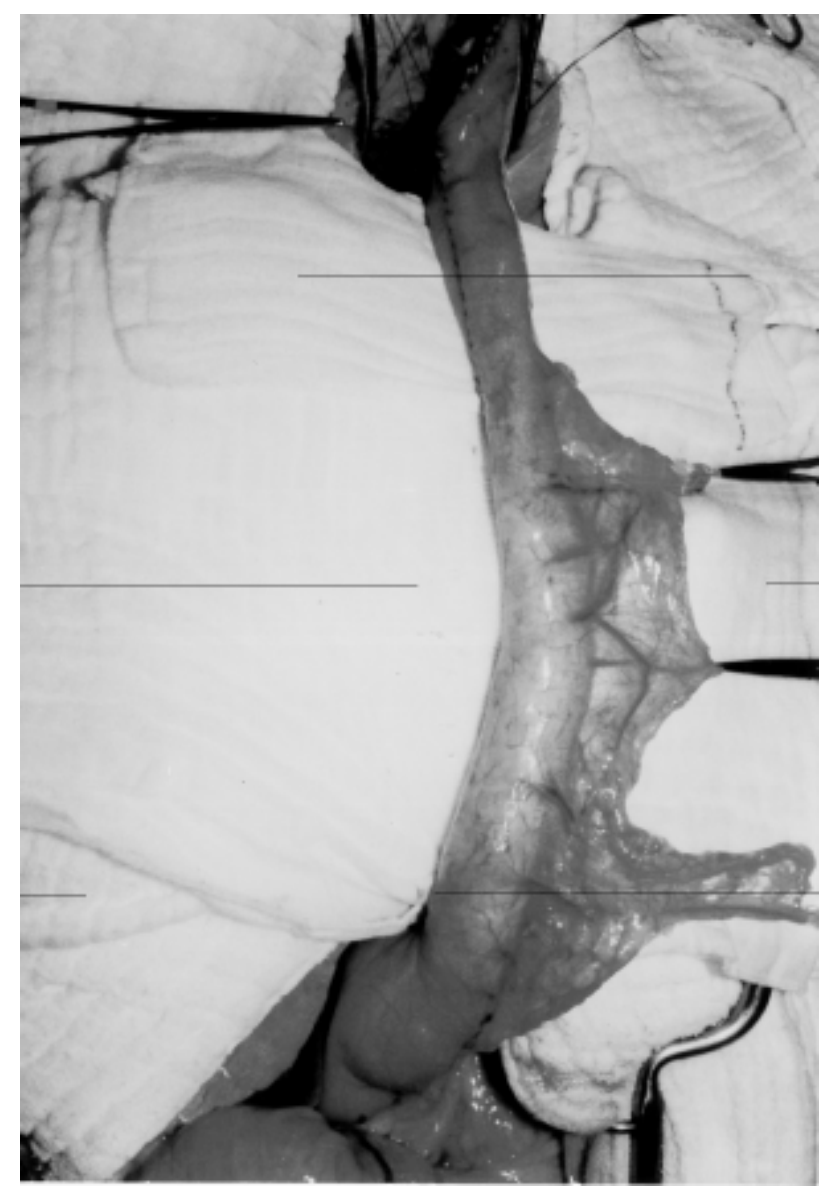

Figura 1 - Tubo gástrico isoperistáltico obtido da grande curvatura.

são cervical para permitir ampla drenagem e o paciente era orientado a deglutir $150 \mathrm{ml}$ (um copo) de água três a quatro vezes por dia para "lavar" o trajeto fistuloso. Com o fechamento da fístula, a dieta por via oral era iniciada, mantendo a sonda nasoenteral por mais três dias.

$\mathrm{Na}$ alta hospitalar, os pacientes eram liberados para ingerir dieta livre, encaminhados para radioterapia e orientados a retornar semanalmente ao ambulatório para avaliar a tolerância ao tratamento radioterápico.

\section{RESULTADOS}

Não houve óbito intra-operatório. Lesão esplênica ocorreu em dois pacientes $(6,6 \%)$, que foi tratada com esplenectomia. Complicações pós-operatórias ocorreram em 21 pacientes (70\%) (Tabela 1); três pacientes (10\%) apresentaram complicações pós-operatórias graves e culminaram com o óbito: um caso devido a tromboembolismo pulmonar e dois devido à infecção pulmonar; os dois últimos apresentavam metástases hepáticas. Treze pacientes $(43,3 \%)$ evoluíram com fístula cervical, geralmente após o sétimo dia pós-operatório; na maioria (11 casos) ocorreu fechamento espontâneo da fístula entre o $14^{\circ}$ 
Tabela 1

Tubo gástrico isoperistáltico no tratamento paliativo do carcinoma irressecável do esôfago - complicações pós-operatórias*

\begin{tabular}{l|c|c}
\hline Complicação & Número & $\%$ \\
\hline Fístula & 13 & 43,3 \\
Estenose & 8 & 26,6 \\
Broncopneumonia & 8 & 26,6 \\
Derrame pleural & 3 & 10,0 \\
Óbito & 3 & 10,0 \\
Atelectasia & 2 & 6,6 \\
Insuficiência renal aguda & 2 & 6,6 \\
Tromboembolismo pulmonar & 1 & 3,3 \\
\hline
\end{tabular}

* Mais de uma complicação ocorreu em um mesmo doente

e o $30^{\circ}$ dia pós-operatório, apenas com cuidados locais. Um paciente $(3,3 \%)$ necessitou de reintervenção no $27^{\circ}$ dia pósoperatório. Refeita a anastomose, novamente ocorreu fistulização, que se resolveu após 13 dias. Outro paciente veio a óbito no $14^{\circ}$ dia pós-operatório em virtude de complicações pulmonares, com fístula aberta. Oito pacientes $(26,6 \%)$ evoluíram com estenose da anastomose esôfagotubo, dos quais quatro tiveram, anteriormente, fístula. Todos os casos evoluíram bem com dilatação endoscópica. A deglutição foi restabelecida em todos os pacientes acompanhados até o momento do óbito, excetuando aqueles que faleceram em virtude de complicações pós-operatórias (três casos). O tempo de internação variou de 12 a 45 dias (média de 18 dias e mediana de 15 dias). Seis pacientes (20\%) abandonaram o seguimento após o término da radioterapia sem sinais de disseminação da doença; para efeito de cálculo, foram considerados falecidos. Vinte e um pacientes vieram a falecer em consequiência da disseminação da doença. A sobrevida variou de dois a 24 meses, com média de 7,9 meses e mediana de 7,5 meses.

Um paciente, após o término da radioterapia, encontrava-se em bom estado geral e, ao novo estadiamento, não apresentava sinais de disseminação; foi realizada esofagectomia por toracotomia direita. O exame anatomopatológico da peça mostrou carcinoma espinocelular residual, infiltrando até adventícia, com margens livres. O paciente evoluiu bem, ganhou 22 quilos, porém 24 meses após a primeira cirurgia, veio a óbito por disseminação neoplásica.

\section{DISCUSSÃO}

A cirurgia e a radioterapia são as principais armas para o tratamento do carcinoma do esôfago ${ }^{12}$. A orientação quase que universalmente adotada é a de ressecar o tumor sempre que tecnicamente possível ${ }^{12}$. Nos casos de câncer avançado, o papel da cirurgia de derivação é controverso, pois pode restaurar a deglutição normal durante a curta sobrevivência do paciente, porém não é destituída de risco ${ }^{12}$. A utilização de tubos gástricos é uma das formas de derivação. O tubo com a grande curvatura do estômago, nutrido pela artéria gastroepiplóica esquerda (anisoperistáltico), foi concebido por Beck e Carrel em $1905^{17}$, é o precursor do tubo gástrico utilizado no ser humano, e foi divulgado a partir de 1951 por Gavriliu ${ }^{18}$ e por Heimlich et al. ${ }^{19}$. A primeira referência ao tubo gástrico nutrido pela artéria gastroepiplóica direita (isoperistáltico) foi feita por Dengel em $1930{ }^{20}$, que atribuiu sua concepção a Rutkowiski ${ }^{20}$; somente em 1979 Postlethwait relata a utilização do tubo gástrico isoperistáltico para transpor o esôfago com neoplasia avançada ${ }^{12,21}$. Em 1989, Speranzini et al. ${ }^{12}$ introduziram esta técnica em nosso meio e a partir de 1990 passamos a utilizá-la.

No presente estudo a mortalidade operatória foi de 10\%; os resultados da literatura são bastante heterogêneos variando entre $0 \%$ e $40 \%$ 2, 6, 12, 21-25. Esta discrepância de resultados provavelmente se deve à falta de uniformidade nas técnicas operatórias utilizadas, diferenças de infra-estrutura hospitalar, diferenças nos critérios de escolha dos pacientes e nos critérios de irressecabilidade e operabilidade (avaliação das condições clínicas) ${ }^{2,23}$. Consideramos irressecáveis tumores com mais de $6 \mathrm{~cm}$ de extensão ao esofagograma ou com sinais de abaulamento ou invasão da árvore respiratória à broncoscopia. Pacientes muito depauperados ou com doenças associadas de difícil compensação foram considerados inoperáveis. Ocorreram dois óbitos no período pós-operatório precoce devido à broncopneumonia (ambos apresentavam metástases hepáticas) demonstrando que talvez estes pacientes não apresentassem condições clínicas adequadas para suportarem o ato operatório. Fisioterapia respiratória e suporte nutricional parenteral ou enteral por sete a dez dias, no período pré-operatório, representam medidas importantes no preparo destes pacientes. Meniconi preconiza suporte nutricional aos pacientes com perda ponderal superior a $15 \%$ em relação ao peso habitual e com níveis séricos de albumina inferior a $3 \mathrm{~g} \%$, até atingirem condições cirúrgicas adequadas ${ }^{6}$.

A morbidade do procedimento não é baixa (Tabela 1). A complicação mais freqüentemente observada foi a fístula esofagocutânea em nível cervical, que ocorreu em 13 doentes $(43,3 \%)$. Na maioria das vezes (11 doentes) ocorreu o fechamento espontâneo da fístula entre o $14^{\circ}$ e e o $30^{\circ}$ dia pós-operatório, apenas com cuidados locais e nutrição enteral por cateter de Dobbhoff. Para obter boa evolução da fístula consideramos importantes as seguintes medidas: ingestão de um copo de água três a quatro vezes por dia para "lavar" o trajeto fistuloso (ajudando na limpeza de muco ou secreção purulenta que algumas vezes se acumula no trajeto) abertura da pele para permitir ampla drenagem e não utilizar curativos oclusivos nem bolsas coletoras que dificilmente aderem à pele da região cervical dificultando a higiene local; colocamos à disposição do doente inúmeras compressas, e ele mesmo realizava a limpeza local tantas vezes quanto precisasse. A literatura mostra uma incidência de fístula que varia de $0 \%$ a $58 \%$; a maioria dos trabalhos mostra incidência maior que $20 \%$,2, 12,23-25. Embora freqüente, a fístula cervical geralmente tem evo- 
lução benigna, com a resolução espontânea ${ }^{2,12}$. Apenas um dos nossos pacientes necessitou de reintervenção para resolução da fístula e outro veio a óbito com a fístula aberta no 14 으 dia pós-operatório.

Vários fatores podem ser apontados como causadores desta fístula: a anastomose é realizada na porção menos irrigada do tubo (isquemia), contaminação exagerada do esôfago principalmente na presença de obstrução, pressão endoluminal esofágica aumentada à deglutição de saliva e disposição em baioneta do eixo esofágico cervical em relação ao eixo do tubo gástrico da grande curvatura passado à região cervical, via retroesternal, favorecendo a compressão da anastomose e estase a este nível ${ }^{6,12}$.

A estenose ocorreu com frequiência nos doentes que apresentavam fístula previamente. Oito dos nossos doentes $(26,6 \%)$ desenvolveram estenose, dos quais quatro após o fechamento da fístula. Todos os casos evoluíram bem com dilatação endoscópica, o que esta de acordo com a literatura ${ }^{2,12}$.

A desnutrição, a idade avançada e o hábito de fumar (freqüente nestes doentes) são responsáveis pela alta incidência de complicações respiratórias ${ }^{2}$ : três pacientes apresentaram derrame pleural que não necessitou nenhum tipo de tratamento especial, dois desenvolveram atelectasia, resolvida com fisioterapia respiratória, oito apresentaram broncopneumonia (dois óbitos) e um evoluiu com embolia pulmonar fatal. Este resultado enfatiza a necessidade de criteriosa avaliação clínica ${ }^{12,23}$, rigoroso preparo pré-operatório (suporte nutricional, compensação das doenças associadas e fisioterapia respiratória), preocupação com um tempo cirúrgico não muito longo (que é atingido com duas equipes bem treinadas atuando simultaneamente e com o uso do grampeador linear cortante) e cuidados intensivos no período pós-operatório.

A deglutição foi restabelecida até o momento do óbito em todos os pacientes, exceto em um que veio a óbito ainda com fístula no $14^{\circ}$ dia pós-operatório

A derivação cirúrgica não modifica a evolução natural da doença e o período médio de sobrevivência varia de cinco a 12 meses $^{2,6,10,12}$. No nosso estudo foi de 7,9 meses.

Na presente casuística, a radioterapia foi eleita como terapêutica complementar. Um dos nossos pacientes apresentava bom estado geral e nenhum sinal de disseminação ao final do tratamento radioterápico; foi submetido então a uma esofagectomia subtotal por toracotomia direita com intenção curativa; teve boa evolução e representou o caso que apresentou o mais longo período de sobrevivência (24 meses). A utilização do tubo gástrico isoperistáltico para a correção da disfagia não impede a adoção da terapêutica com tentativa curativa; podendo representar uma das etapas de um plano terapêutico ${ }^{12}$.

Concluímos que nos pacientes portadores de carcinoma irressecável do esôfago, a derivação da obstrução através de um tubo gástrico isoperistáltico atinge seu objetivo primeiro que é aliviar a disfagia, na maioria dos casos, até o momento do óbito; a morbidade é elevada, porém a maioria das complicações tem evolução benigna; a mortalidade é aceitável levando-se em conta a gravidade e a evolução devastadora do carcinoma do esôfago.

\begin{abstract}
The goal of the treatment in patients with advanced esophageal carcinoma is to alleviate dysphagia. Background: The objective of this study is to report the authors' experience with gastric esophageal bypass using isoperistaltic gastric tube followed by radiotherapy, in the treatment of patients with unresectable esophageal carcinoma. Method: From 1990 to 1999, 30 patients underwent bypass surgery. Twenty four patients (80\%) were male and six (20\%) female, age ranged from 27 to 69 years, with a mean age of 49.3 years. Diagnosis was confirmed by esophagogastroduodenoscopy and biopsy in all patients. Those with tumors greater than $6 \mathrm{~cm}$ at esophagography or with signs of invasion of the respiratory sistem at bronchoscopy were considered unresectable. Bypass surgery was carried out after medical assessment and preoperative evaluation. Surgery was performed by two teams, one at the cervical area and another at the abdominal area. Surgical time ranged from 3 to 4 hours. After hospital discharge, patients were referred to radiotherapy. Results: There was no operative mortality. Postoperative mortality was $10 \%$, one case of pulmonary thromboembolism and two cases of pneumonia. Thirteen patients (43.3\%) developed cervical anastomotic leak and eleven had spontaneous resolution; one required a new operation and another patient died at postoperative day 14, with an open fistula. Eight patients (26.6\%) had anastomotic stenosis, and all of them had a good outcome with endoscopic dilation. Deglutition was reestablished in all patients until death, excluding those who died as a result of postoperative complications (3 cases). Hospitalization time ranged from 12 to 45 days and mean survival rate was 7.9 months. Conclusions: We concluded that, in patients with esophageal unresectable carcinoma, obstruction bypass using an isoperistaltic gastric tube alleviates dysphagia symptoms until death. Morbidity is high, even though most of the complications have a benign outcome. The mortality rate is acceptable taking into consideration the severity of esophageal carcinoma.
\end{abstract}

Key Words: Esophageal carcinoma; Isoperistaltic gastric tube; Correction surgery. 


\section{REFERÊNCIAS}

1. Altorki NK, Girardi L, Skinner DB. En bloc esophagectomy improves survival for stage III esophageal cancer. J Thorac Cardiovasc Surg 1997; 114: 948-56.

2. Alcantara PSM, Spencer-Netto FAC, Silva Junior JF et al. Gastro esophageal isoperistaltic bypass in the palliation of irresectable thoracic esophageal cancer. Int Surg 1997; 82: 249-53.

3. Khandelwal M. Palliative therapy for carcinoma of the esophagus. Compr Ther 1995; 21: 177-83.

4. Ahlquist DA, Gastout CJ, Viggiano TR et al. Endoscopic laser palliation of malignant dysphagia: prospective study. Mayo Clin Proc 1987; 62: 867-74.

5. Maunoury V, Brunetand JM, Cochelard D et al. Endoscopic palliation for inoperable malignant dysphagia: long term follow up. Gut 1992; 33: 1602-7.

6. Meniconi MTM. Estudo prospectivo da aplicação de tubo gástrico de grande curvatura, isoperistáltico, no tratamento da neoplasia avançada de esôfago. Análise de 50 casos. Tese (Doutorado). São Paulo. Faculdade de Medicina da Universidade de São Paulo 1997; 8p.

7. Reed CE. Comparison for different treatments for unresectable esophageal cancer. World J Surg 1995; 19: 828 35.

8. Tomizou LA, Rampton D, Bown SG. Treatment of malignant strictures of the cervical esophagus by endoscopic intubation using modified endoprotheses. Gastrointest Endosc 1992; 2: 158-64.

9. Malafaia O. Experiência de 15 anos com o tratamento paliativo do câncer do esôfago através da tunelização esofágica. Rev Col Bras Cir 1986; 13: 211- 5.

10. Domene CE. Tunelização esofágica no contexto dos métodos de tratamento paliativo do câncer do esôfago e da cardia. Tese (Doutorado). São Paulo. Faculdade de Medicina da Universidade de São Paulo 1989; 152p.

11. Vermeijden JR, Bartelsman JF, Fockens P et al. Self expanding metal stents for palliation of esophagocardial malignancies. Gastrointest Endosc 1995; 1: 58-63.

12. Speranzini MB, Fujimura I, Pires PWA et al. Esofagoplastia com tubo gástrico isoperistáltico em derivação no tratamento do câncer do esôfago torácico: estudo de 13 casos. Rev Ass Med Brasil 1989; 35: 91-8.

13. Webster MW, Carey LC, Ravitch MM. The permanent gastrostomy - use of the gastrointestinal anastomotic stapler. Arch Surg 1975; 110: 658-60.

14. Giménez ME, Anzorena S, Cerisoli F, Caro C, Buabse F, Ferraina PA. Gastrostomia percutánea. Rev Argent Cir 1999; 76:106-12.

15. Sharma D, De Silva J, Saxena A et al. In-continuity fundic bypass for palliation in unresectable carcinoma of the oesophagus: a previously unreported procedure. Dig Surg 2000; 17:306-9.

16. De Moura EGH. Tratamento paliativo do câncer do esôfago por endoprótese e tubo gástrico isoperistáltico. Tese (Doutorado). São Paulo. Faculdade de Medicina da Universidade de São Paulo 2000; 90p.

17. Beck C, Carrel A. Demonstration of specimens illustrating a method of formation of a prethoracic esophagus. Ill Med J 1905; 7: 463-4.

18. Gavriliu D. Aspects of esophageal surgery. Curr Probl Surg $1975 ; 12: 36-64$
19. Heimlich HJ, Winfield JM. The use of a gastric tube to replace or bypass the esophagus. Surgery $1955 ; 549-59$.

20. Dengel L. Plastic restoration of the esophagus. Ann Surg 1930; 92: 51-6.

21. Postlethwait RW. Technique for isoperistaltic gastric tube for esophageal bypass. Ann Surg 1979; 189: 673-6.

22. Postlethwait RW. Complications and deaths after operations for esophageal carcinoma. J Thorac Cardiovasc Surg 1983; 85: 827-31.

23. Corsi PR, Campos T, Pinto MCC et al. Tubo gástrico isoperistáltico no tratamento paliativo do carcinoma do esôfago. An Paul Med Cir 1999; 126: 106-10.

24. Brandalise NA, Andreollo NA, Leonardi LS et al. Utilização do tubo gástrico na reconstrução do trânsito digestivo em neoplasias de esôfago e junção esôfago gástrica. Rev Col Bras 1985; 12: 152-5 .

25. Orringer MB. Substernal gastric bypass of the excluded esophagus. Results of an ill - advised operation. Surgery 1984; 96: 467-70.

Endereço para correspondência:

Dr. Alexandre Cruz Henriques

Rua Mediterrâneo, 928

09750-420 - São Bernardo do Campo-SP

e-mail:rmfaure@ig.com.br 